\title{
Probing dynamics of fusion reactions through cross-section and spin distribution measurement
}

\author{
Maninder Kaur ${ }^{1,2} *$, B.R. BeherA ${ }^{1}$, Gulzar Singh $^{1}$, \\ VARINDERJit Singh ${ }^{1}$, N. MAdHAVAN ${ }^{3}$, S. MURAlithar $^{3}$, S. NATH $^{3}$, \\ J. Gehlot ${ }^{3}$, G. Mohanto ${ }^{3}$, Ish Mukul ${ }^{3}$, D. Siwal ${ }^{4}$, M. Thakur ${ }^{1}$,

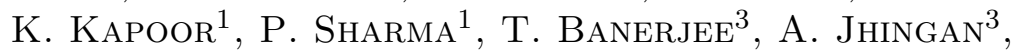 \\ T. Varughese ${ }^{3}$, Indu Bala ${ }^{3}$, B.K. Nayak ${ }^{5}, A$. Saxena $^{5}$, \\ M.B. ChatterueE ${ }^{5}$ and P.D. Stevenson 6
}

1 Department of Physics, Panjab University, Chandigarh - 160014, India

2 Department of Physics, SGTB Khalsa College, Anandpur Sahib - 140118, Punjab, India

3 Inter University Accelerator Centre, Aruna Asif Ali Marg, New Delhi 110067, India

${ }^{4}$ Department of Physics and Astrophysics, University of Delhi - 110007, India

5 Nuclear Physics Division, Bhabha Atomic Research Centre, Mumbai 400085, India

${ }^{6}$ Department of Physics, University of Surrey, Guildford, Surrey, GU2 $7 \mathrm{XH}, \mathrm{UK}$

\begin{abstract}
Present work aims to explicate the effect of entrance channel mass asymmetry on fusion dynamics for the Compound Nucleus ${ }^{80} \mathrm{Sr}$ populated through two different channels, ${ }^{16} \mathrm{O}+{ }^{64} \mathrm{Zn}$ and ${ }^{32} \mathrm{~S}+{ }^{48} \mathrm{Ti}$, using cross-section and spin distribution measurements as probes. The evaporation spectra studies for these systems, reported earlier indicate the presence of dynamical effects for mass symmetric ${ }^{32} \mathrm{~S}+{ }^{48} \mathrm{Ti}$ system.
\end{abstract}

*E-mail: manisaini153@gmail.com 
The CCDEF and TDHF calculations have been performed for both the systems and an attempt has been made to explain the reported deviations in the $\alpha$-particle spectrum for the mass symmetric system.

\section{Introduction}

The Compound nucleus $(\mathrm{CN})$ populated at high excitation energy and angular momentum can undergo decay through fission or emission of different light particles. The study of these light particles and their analysis in terms of statistical model gives important information about the properties of the CN. Though a large amount of such data exists in the literature, but many of these studies show anomalous results for the mass symmetric systems $[1-3]$ that could not be explained in terms of the statistical model predictions. These deviations from the statistical model predictions were explained through the modification of important ingredients of the statistical model codes such as spin dependent level density, lowering the level density parameter or using lower values of $l$ contributing to fusion. However the simultaneous analysis of other variables such as spin distribution, cross-section measurement, angular distribution measurements etc. should also be taken into account to have a better understanding of fusion dynamics. With this motivation we have performed the spin distribution and the cross-section measurements for two systems ${ }^{16} \mathrm{O}+{ }^{64} \mathrm{Zn}$ (mass asymmetric) and ${ }^{32} \mathrm{~S}+{ }^{48} \mathrm{Ti}$ (mass symmetric) populating the same $\mathrm{CN}\left({ }^{80} \mathrm{Sr}\right)$ for which the evaporation spectra studies have already been performed $[1,2]$. These studies also report the existence of deviations for the symmetric system. Present measurements have been performed using 15 UD Pelletron accelerator and Heavy Ion Reaction Analyzer (HIRA) facility at Inter University Accelerator Centre (IUAC), New Delhi. The details of these measurements and analysis procedure have been described elsewhere [4].

\section{Analysis and results}

The evaporation residues (ERs) populated during the reaction were separated from beam-like background using HIRA. For this, a two-dimensional spectrum was generated using energy loss and Time of Flight (TOF) of ERs. The value of transmission efficiency of different ERs through HIRA was estimated by $\gamma$-ray co-incidence method and using the TERS code. The experimental cross-sections thus obtained were compared with CCDEF and TDHF [5] calculations. For both the systems the experimental cross-sections 


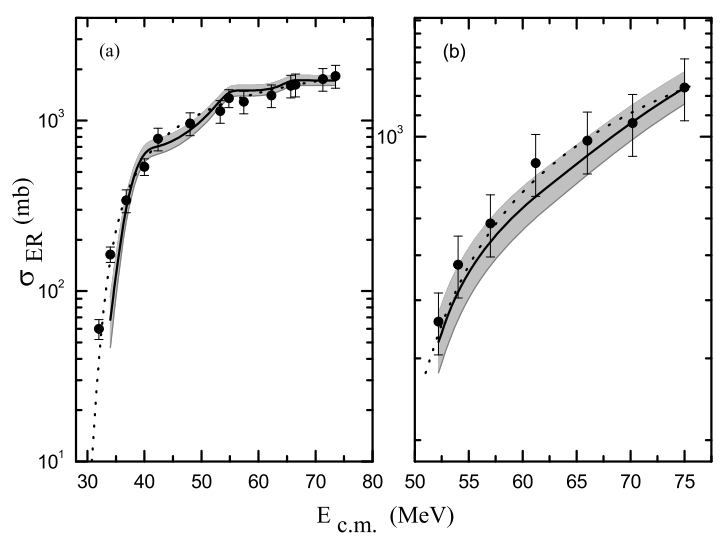

Figure 1: The comparison of the experimental cross-sections (solid circles) with the theoretical calculations for (a) ${ }^{16} \mathrm{O}+{ }^{64} \mathrm{Zn}$, (b) for ${ }^{32} \mathrm{~S}+{ }^{48} \mathrm{Ti}$. The solid line corresponds to the TDHF calculations and shaded area represents the uncertainty in cross-sections obtained from the TDHF calculations. The dotted lines corresponds to the CCDEF calculations

were in agreement with these calculations as shown in fig. 1. However, the fusion time scales obtained using the TDHF calculations, at highest energies, were found to approach the decay times showing that the fusion time scales are elongated that may lead to pre-equilibrium emission resulting in the modification of the $\mathrm{CN}$ spin distribution.

So to have a better picture of the contribution of various partial waves to the fusion process, we have also performed the ER-gated spin distribution measurements. Van der Werf's prescription was used for unfolding the $\gamma$-multiplicity distributions from $\gamma$-fold distributions. $\gamma$-multiplicity distributions were converted to spin distribution by assuming that average spin carried away by each non-statistical $\gamma$-ray is 1.6 and incorporating the corrections from the spin carried away by the light particles. Using the experimental cross-sections, the $\mathrm{CN}$ spin distributions were obtained. These were compared with CCDEF calculations. It was observed that for the symmetric system, the contribution of the higher partial waves towards fusion goes on decreasing with the increase in energy (fig. 2(a)). These observations are in agreement with the calculations of fusion time scales extracted using TDHF calculations. These observations were not reflected in the cross-section measurements as the cross-sections correspond to the zeroth moment of the spin distribution, so there can be many shapes of the spin distribution, reflecting very different physical processes involved, but may yield the same fusion cross-section. The experimental spin distributions, for ${ }^{32} \mathrm{~S}+{ }^{48} \mathrm{Ti}$ at $E_{\text {lab }}$ $=125 \mathrm{MeV}$, were give as an input to the PACE code and the generated $\alpha$ particle spectrum was found to be in excellent agreement with experimental spectrum (fig. 2(b)), thus explaining the deviations reported earlier. 


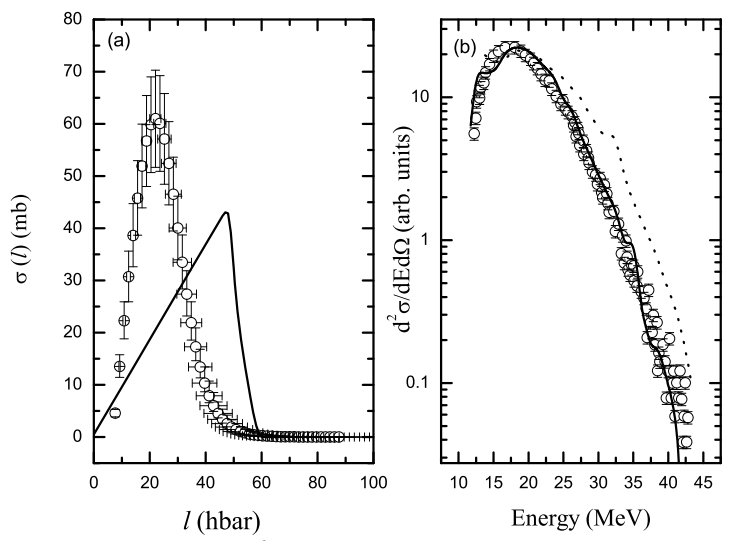

Figure 2: (a) The comparison of the experimental spin distributions (circles) with CCDEF calculations (solid line) for ${ }^{32} \mathrm{~S}+{ }^{48} \mathrm{Ti}$ at $E_{l a b}=125 \mathrm{MeV}$. (b) The explanation of experimental $\alpha$-particle spectrum (circles) by the spectrum generated (solid line) using experimental spin distribution in PACE. The dotted line corresponds to default calculations using PACE.

\section{Conclusions}

The measured experimental cross-sections were well explained by the CCDEF and TDHF calculations. However, the comparison of experimental spin distributions with the the CCDEF calculations indicate the effect of entrance channel mass asymmetry on fusion dynamics and the deviations in the experimental $\alpha$-particle spectrum for the system ${ }^{32} \mathrm{~S}+{ }^{48} \mathrm{Ti}$ were reasonably explained. Therefore, we conclude that the shape of the spin distribution can act as a sensitive tool for the investigation of fusion dynamics.

\section{Acknowledgements}

We thank the accelerator crew of IUAC for providing beams of excellent quality throughout the experiments. One of the authors (M.K.) would like to thank University Grants Commission (UGC) for the financial assistance.

\section{References}

[1] J. Kaur et al., Phys. Rev. C 70, (2004) 017601.

[2] Ajay Kumar et al., Phys. Rev. C 68, (2003) 034603.

[3] M. Kaur et al., Phys.Rev. C 89, (2014) 034621.

[4] M. Kaur et al., DAE Symp. Nucl. Phys. 58, (2013) 434; 59, (2014) 350.

[5] J. A. Maruhn et al., Comput. Phys. Commun. 185,(2014) 2195. 